\title{
Discussions on the Prediction of Fatigue Life of Mechanical Structure and the Reliability Method
}

\author{
Guoli Sun \\ Jiangsu Province Xuzhou Technician Institute, Xuzhou, Jiangsu, 221000, China
}

Keywords: Mechanical structure; Fatigue life; Reliability method; Life prediction

\begin{abstract}
In this paper, the main methods of fatigue life prediction of mechanical structure are discussed. The necessity of predicting the fatigue life of mechanical structure is presented from the point of view of fracture mechanics. The damage tolerance is introduced, and the safety evaluation and analysis of mechanical structure fatigue life prediction the outline design is given. Aiming at the rotor type mechanical structure product, the reliability design problem of the predetermined service life of the mechanical structure in the design stage is discussed. Based on the probability distribution of fatigue strength under the specified life of $\mathrm{P}-\mathrm{S}-\mathrm{N}$ curve, the probability distribution function of mechanical structure under different working loads is obtained through experiments according to the theory of stress intensity interference, the mechanical structure product design the calculation method, as well as in the specified life of the mechanical junction of high reliability design.
\end{abstract}

\section{Introduction}

For the fatigue life prediction and reliability research of mechanical structure, it has very important application significance and research value in many cutting-edge manufacturing fields, including the advanced industries such as aerospace, high-speed train and shipbuilding, in terms of safety and reliability, the use of general machinery and equipment also plays an important role. Relevant foreign statistics show that the damage caused by fatigue accounts for a large proportion of the damage of mechanical structural parts, about $50 \%$ to $90 \%$. As a result of the mechanical structure caused by the destruction of the problem has not been conducted a comprehensive survey, but compared with developed countries, the shorter life of similar mechanical products. In particular, some heavy machinery and equipment for loading and unloading, the load-bearing components are steel structure, in the long-term loading and unloading process, the contrast between the larger external loads continue to cycle, the mechanical structure of the cracks, expansion, or even sudden rupture damage the possibility of greater. During the actual use of mechanical products, the destructive problems caused by structural fatigue are relatively common, the fatigue life of mechanical structure is correctly estimated and predicted, the probability of mechanical product safety is evaluated, and the reliability design method of mechanical structure is studied, The safe operation of the equipment using the system, so that the mechanical structure of the reliability of the firmware further improve the index, of great significance.

Due to the different forecasting principles of mechanical components, the life prediction methods of mechanical products can be divided into two types: cumulative damage method and characteristic parameter method. Cumulative damage method based on failure physics develops rapidly in the mechanical life prediction, and life prediction theory and method based on fatigue analysis have become more and more mature in aerospace technology. Factors that affect the performance of rotating machinery, leading to its performance degradation include: aging of organic materials, creeping of metal materials, moving parts wear and fatigue, these factors are not independent of the development of damage, a variety of damage factors occur under certain conditions Interaction. In the study of the interaction between various damage factors, the problem of life span of mechanical mechanism was considered. Considering that the degradation failure model can comprehensively analyze various damage factors, the model can make use of the 
degradation data to provide more process information of life development. In related researches, the failure mechanism of mechanical structure degradation is constructed by using the normal stochastic process model. The mechanical structure of the firmware life test and its parameters estimation, degradation failure model, so as to further explore the mechanical life prediction method. By analyzing the distribution of profitability during the operation of mechanical structure, the life expectancy is obtained.

After the mechanical product is shaped, the fatigue part of the mechanical structure and its fatigue life can be finally determined. During the experiment, some problems will be found. According to these problems, the original design needs to be modified. Such experiments modifying the design scheme re-experimenting and then modifying the original design will lead to the prolongation of the product development cycle, resulting in the product Research and development costs are too high. Therefore, in the case of the specified life, to study the reliability of the mechanical structure method is the current study of the fatigue life of the mechanical structure of the urgent need to be addressed.

\section{Mechanical Structure Fatigue Life Prediction Method}

The mechanical fatigue life prediction methods include: nominal stress method, local stress and strain method, damage tolerance method, fatigue reliability method.

\subsection{Nominal stress method}

The basic parameters of the method for the nominal stress, calculated as S-N curve. According to different design life, the method can be divided into finite and infinite life design; according to different nominal stress parameters, divided into stress amplitude and stress ratio method. The nominal stress method to predict the fatigue life of the mechanical structure of the basic steps are as follows:

Step1: In the typical working time course, the statistic counting method is used to get the stress circulation status of the fatigue prediction and analysis position of the mechanical structure, and the load spectrum and stress spectrum of the mechanical structure are determined. Stress cycle can be detected on-site structure strain to get the stress spectrum, but also can be a typical working state of the calculation and analysis;

Step2: Considering the influence coefficient of various factors, combined with the S-N curve of the steel material, and considering various influence coefficients, the S-N curve of the mechanical structure firmware is obtained;

Step3: Analyze the fatigue strength of the mechanical structure according to the Miner's rule or the modified Miner's rule. The comparison of cumulative damage: Miner's law belongs to the linear cumulative damage, easy to use, but ignores the residual stress, loading sequence on the mechanical structure caused by the mechanical structure of the fatigue life prediction results and the actual life of a larger difference; Miner's law belongs to the non-linear rule, which can improve the accuracy of the predictive analysis. The commonly used nonlinear rules include: Corten-Dolan index damage method and Marco-Starkey damage curve method;

Step4: Based on the accumulated damage theory, the fatigue life of the mechanical structure is predicted. The remaining life of the mechanical structure of the calculation method: the total damage as a basis for crack generation, cracks calculated to form the mechanical life of the structure, remove the mechanical structure of the equipment has been used in time to predict the remaining life of the mechanical structure;

Step5: Verification of the mechanical structure of the fatigue test. Generally used in the equipment used for fatigue analysis, due to the large-scale mechanical institutions of some large-scale equipment modeling, fatigue test proved difficult to complete. The current common practice is: in the mechanical structure of the fatigue life prediction period, regular safety inspection, monitoring equipment, dangerous points of fatigue, to ensure the safety of the use of mechanical structure. In the mechanical structure of fatigue life calculation, the use of nominal stress method has many advantages: the theory and on-site testing is simple and easy to carry out stress analysis. 
However, the preconditions required by this method are that there are no defects in the mechanical structure material, no cracks in the mechanical structure and material, and the total life of the mechanical structure minus the working time is taken as the number of stress cycles; and the actual working mechanical structure Equipment, mechanical structure of the initial internal cracks or defects appear to belong to the normal phenomenon, these cracks may be macro or micro defects; In addition, many users do not leave a detailed and accurate historical records of fatigue life analysis equipment, historical records, these factors All have a serious impact on the accuracy of prediction and prediction of the fatigue life of the mechanical structure by using the nominal stress method. The life expectancy of a mechanical structure and the location of a nominal stress method are shown in Figure 1 below.

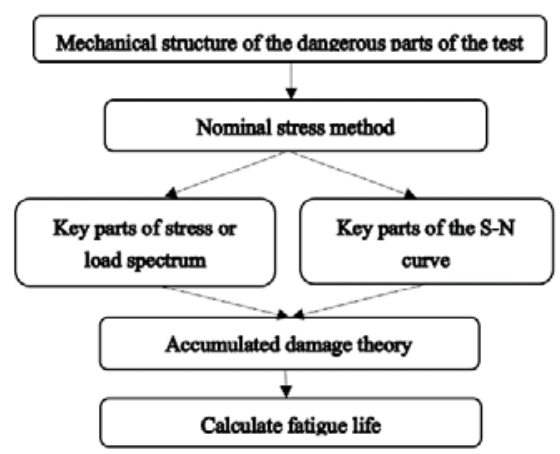

Figure 1 mechanical structure fatigue life prediction steps

\subsection{Fatigue life reliability method}

The mechanical structure of the external load, fatigue performance and other data on the mechanical structure of the fatigue damage to make a limited probability to ensure that the mechanical structure and the steel structure within a certain range of material integrity. In the method of predicting the fatigue life of mechanical structure, the nominal stress method is used to estimate the life expectancy of the sloped part of the S-N curve to obtain the total life of the mechanical structure. The total life of the mechanical structure is composed of the life of crack propagation and the life of crack formation.

\subsection{Damage tolerance method}

The method is based on fracture mechanics. The basic principle: Recognizing the existence of initial crack defects, the mechanical structure will crack under the external load expansion, according to the nature of this crack propagation, fatigue life prediction. Fracture mechanics is the theoretical basis of the damage tolerance method, the determination of fracture toughness and the calculation of fatigue crack growth rate, as well as the non-destructive testing technique is the basic method of damage tolerance method. The initial defect or crack mechanical structure as a condition to predict the remaining life of the mechanical structure to ensure safe use.

Stress Intensity Factor: When mechanical crack exists, the theoretical stress of crack tip is infinite. The stress intensity factor $\mathrm{K}$ expression coefficient is needed. The $\mathrm{K}$ value characterizes the stress field in the vicinity of the crack to determine whether the instability damage has occurred. The stress intensity of type I(open type), type II(sliding open type) and type III(tear open type) crack tip are respectively expressed by stress intensity factors $K_{1} 、 K_{2} 、 K_{3}$; the most mature type I is studied.

In general, the stress intensity factor $\mathrm{K}$ can be expressed as:

$$
\mathrm{K}=\mathrm{F} \sigma \sqrt{\pi a}
$$

Where $\mathrm{F}$ denotes the coefficient that determines the crack shape, crack location, shape and loading method, which may be a function of constant or a (crack size).

Fracture Toughness: The stress intensity factor at which brittle fracture occurs, which is the critical value of the stress intensity factor, expressed as $K_{c} . \mathrm{K} \geq K_{c}$ is the basis for fracture diagnosis of fracture Mechanics. 
Plane strain fracture toughness $K_{1 c}$ : Critical stress intensity factor of type I crack under plane strain condition, $K_{1 c}$ reflects the material toughness, which means the minimum value of fracture toughness is reached. Under plane strain condition, $K_{1} \geq K_{1 c}$ means fracture criterion, $K_{1 c}$ can be determined by experiment, and $K_{1 c}$ value of commonly used materials can also be found in the manual.

Fatigue crack growth rate. Denoted by $\mathrm{da} / \mathrm{dN}$ is a function of the range of stress intensity factors.

Estimated remaining life. Finite element analysis of the mechanical structure is performed to find out the key locations where fatigue analysis is needed and to determine the critical crack size, initial crack, stress intensity factor and fatigue crack growth rate at the analysis point. Then, the residual life prediction of the mechanical structure can be carried out.

\subsection{Local stress-strain method}

This mechanical structure fatigue life prediction method is developed on the basis of low cycle fatigue, local stress in the stress concentration and local stress are the basic design parameters. The method is mainly used to predict the fatigue life of a single part. For large and complex structures, it is more difficult to accurately analyze the stress and strain.

\section{Fatigue Life Prediction of Mechanical Structure Based on Flaw Detection}

Based on the defect detection of mechanical structure fatigue life prediction, the main components include: (1) acoustic emission detection device. Mainly used for mechanical cracking and crack growth of materials for dynamic monitoring, detection and positioning; (2) ultrasonic flaw detection device. It is mainly used to detect, locate, evaluate and diagnose the interior of the structure, including various internal defects such as weld, inclusions, cracks and pores. (3) Stress and strain measurement device. For mechanical structure of stress and strain dynamic monitoring and measurement; (4) Mechanical fatigue life prediction analysis and evaluation design. Fracture mechanics as the theoretical basis for the mechanical structure of the fatigue life prediction and analysis methods, the overall design of the system module composition as shown in Figure 2 below.

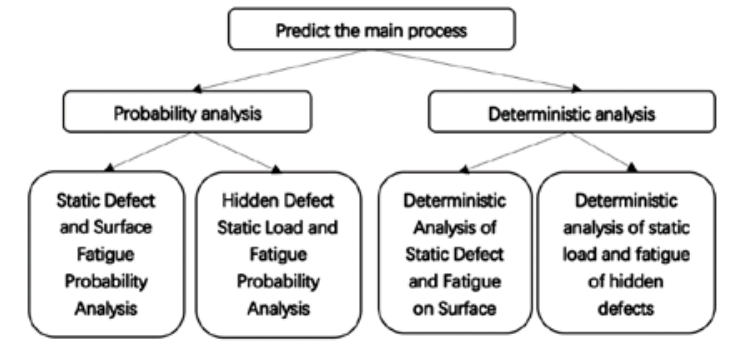

Figure 2 Mechanical structure of the fatigue life prediction analysis and evaluation of design components

In the prediction and analysis of the fatigue life of mechanical structure, combined with the finite element calculation, the key position of fatigue failure is determined, the detection of structural defects is confirmed, the dynamic stress detection (to determine the stress spectrum) can accurately predict the fatigue life of the mechanical structure Assessment, prediction, in order to determine a reasonable safety inspection cycle, to ensure the safety of machinery and equipment during use.

\section{Mechanical Structure Fatigue Reliability Design and Research}

The reliability of the mechanical structure design, the three design indicators are: external load, fatigue strength and the true size of the structural components, they are considered to meet the specific probability distribution of statistical variables; mechanical structure safety life prediction , First the actual dimensions of the mechanical structure and the structural form are to be determined and the mechanical and mechanical dimensions are to be obtained by assigning the lifetimes $\mathrm{N}^{*}$ for the unsafe parts of the mechanical structure for a given degree of reliability in the case where the 
probability of fatigue stress magnitude and the probability of fatigue strength are known, the structure of the structural member, the mechanical size, and the like can be determined.

The probability distribution function of the fatigue stress amplitude of the mechanical structure is denoted by $f(s)$, and the probability distribution function of the fatigue strength of the component under the specified life $\mathrm{N}^{*}$ is expressed as $\mathrm{g}(\mathrm{R})$. According to the stress intensity interference model, when $\mathrm{R}>\mathrm{s}$, When the fatigue strength is higher than the load amplitude stress, in order to ensure the mechanical structure components are not damaged, then the load amplitude stress range $\left(s_{1}-\frac{1}{2} d_{s}, s_{1}+\frac{1}{2} d_{s}\right)$, the reliability $\mathrm{dR}$ can be expressed as:

$$
\mathrm{dR}=\mathrm{f}\left(s_{1}\right) \mathrm{ds} \int_{s_{1}}^{\infty} g(\sigma) d \sigma
$$

Assuming that the fatigue amplitude stress and fatigue strength of mechanical members are two independent random variables for a given life span of $\mathrm{N}^{*}$, and there is no correlation between them, no matter what the fatigue amplitude stress s takes, the fatigue strength $\mathrm{R}$ Must be greater than the probability of $\mathrm{s}$, therefore, the reliability of mechanical components $\mathrm{R}$ can be expressed by the following formula:

$$
\mathrm{R}=\int_{-\infty}^{\infty} f(s)\left(\int_{s}^{\infty} g(\sigma) d_{\sigma}\right) d_{s}
$$

Therefore, it is necessary to know the fatigue amplitude stress distribution $f(s)$ and the fatigue strength probability distribution $g(R)$ of the mechanical structure when designing the reliability of the mechanical structural components under the specified mechanical structure life.

\subsection{Fatigue strength probability distribution of mechanical structure under specified life span}

In designing the reliability of a mechanical mechanism for a given lifetime, it is necessary to know the fatigue strength distribution of the mechanical structural material for a given lifetime, either directly from the experiment or from the fatigue $\mathrm{P}-\mathrm{S}-\mathrm{N}$ curve. With $\mathrm{N}$ fixed, resolving the $\mathrm{P}-\mathrm{S}$ curve yields the fatigue strength distribution of the mechanical structure for the specified lifetime at the specified lifetime. The data obtained by the two methods are compared and the results are basically the same. The experimental method referred to herein refers to the lifting and lowering method in the fatigue experiment. In practical engineering applications, the distribution of fatigue strength under specified lifetimes is generally obtained experimentally.

\subsection{The expression of probability distribution of the external load stress amplitude of mechanical components}

To obtain the probability distribution of the stress amplitude of the external load of the mechanical structural components, the engineering components are needed and simulated in the actual work to get the probability distribution, which lays the foundation for the reliability design of the mechanical structure. Normal distribution, exponential distribution, logarithmic normal distribution, Weibull distribution are commonly used forms of stress amplitude distribution. In the following, taking the normal distribution and exponential distribution as an example, the specific form is given.

Normal distribution: $\mathrm{f}(s)=\frac{1}{S_{s} \sqrt{2 \pi}} e^{-\frac{1}{2}\left(\frac{S-\mu_{s}}{S_{s}}\right)^{2}} ;$ index distribution: $\mathrm{f}(x)=\lambda e^{-\lambda_{s} s}$.

According to the stress intensity interference model, the mechanical structure reliability is solved. The common methods are: graphic method, analytic method, numerical integration method and Monte Carlo method.

\section{Conclusions}

No defects and cracks are the prerequisites of the nominal stress method, but all kinds of cracks are inevitably existed in the real mechanical structure, resulting in a big difference between the prediction results of the fatigue life and the actual situation. The principle of fracture mechanics is the theoretical basis of the damage tolerance method. By detecting the initial crack of the current 
mechanical structure and solving the fatigue crack growth rate, the fatigue life of the mechanical structure can be accurately predicted, which is a relatively effective method in the fatigue life prediction of the mechanical structure. According to the design of the reliability of the mechanical structure under the specified life, the probability distribution of the fatigue strength of the mechanical structure under the specified life can be determined according to $\mathrm{S}-\mathrm{N}$ and $\mathrm{P}-\mathrm{S}-\mathrm{N}$ curves. By studying the safety and reliability of the design of a single mechanical firmware, in order to further study the large reliability of the design laid the foundation.

\section{References}

[1] Zhao Shaoyu. Anti-fatigue design. Beijing: Mechanical Industry Press, 1995.

[2] Dongyue Xiang, Gao Zengliang. A review of fatigue life prediction methods [J]. Large Castings and Forgings, 2006, (3): 39-41.

[3] Liu Weixin. Mechanical Reliability Design [M]. Beijing: Tsinghua University Press, 1996.

[4] Yan Chu-liang, Wang Zhi. Study on fatigue per-formance test for 25 steel in fresh water [J]. Trans-actions of the Chinese Society of A gricultural Ma-chinery, 2002, 33 (5): 89- 92.

[5] Wang Haidong, Zheng Yu, Li Jianqiang. Research on the Reliability of Aircraft and Engine Structure Based on Probabilistic Damage Tolerance Design. Mechanical Design and Manufacturing, 2005 (10): 130-131

[6] Zhao Hu, Liu Ching-qing. Determination of the service life of the safety of port cranes' metal structure. Hoisting and transport machinery, 2006 (8): 74-76

About the Author: Guoli Sun (1981-), male, Jiangsu people, Master's degree, Deputy title. 